\title{
Implementation of Infection Control Practices to Manage Hospital Acquired Infections
}

\author{
T. Jayasree and Mustafa Afzal* \\ Department of Microbiology, CARE Hospitals, Exhibition Grounds Road, Nampally, Hyderabad, \\ Telangana - 500 001, India.
}

\begin{abstract}
Hospital-acquired infections affect millions of patients worldwide each year and result in massive preventable healthcare costs. Many of the organisms that cause these infections survive on routine healthcare equipment and are easily transmitted from patient to patient when healthcare professionals and facilities do not comply with infection prevention and control practices. They are particularly dangerous for high-risk patients and patients in intensive care units. Hence the present study involved the implementation of an infection control protocol to reduce these nosocomial infections. Guidelines for infection control in our hospital setting were formulated by the infection control team in the year 2010. These guidelines were periodically revised to improve the compliance of infection control measures. Additionally, regular auditing of the prescribed antibiotic further minimized the high end and reserved antibiotic use thereby further reducing the nosocomial infection rate. Data regarding the infection rates were collected from the intensive care units and were calculated for the site-specific nosocomial infection. It is observed that $\mathbf{1 7 . 6 \%}$ reduction in the nosocomial infection rate was over a period of 7 years. Additional analysis of the site-specific nosocomial infection rate revealed a 5.2, $12.2,10.5$, and $5.7 \%$ reduction in ventilator-associated pneumonia, catheter-related urinary tract, bloodstream, and surgical site infection rate. The results of the present study showed an improved compliance rate with the infection control guidelines which facilitated the control of nosocomial infection rates thereby reducing the hospital costs, total patient days and the mortality rate.
\end{abstract}

Keywords: Infection control; Acquired infections; Hospital; Nosocomial infection; Bacterial pathogens.

\footnotetext{
*Correspondence: drmustafaafzal@gmail.com

(Received: 10 July 2018; accepted: 31 October 2018)

Citation: T. Jayasree and Mustafa Afzal, Implementation of Infection Control Practices to Manage Hospital Acquired Infections, J Pure Appl Microbiol., 2019; 13(1):591-597 doi: 10.22207/JPAM.13.1.68

(c) The Author(s) 2019. Open Access. This article is distributed under the terms of the Creative Commons Attribution 4.0 International License which permits unrestricted use, sharing, distribution, and reproduction in any medium, provided you give appropriate credit to the original author(s) and the source, provide a link to the Creative Commons license, and indicate if changes were made.
} 


\section{INTRODUCTION}

A major safety concern not just for the patients but also for the healthcare providers is hospital-acquired infections (HAls), otherwise known as nosocomial infections. "Nosocomial" is the phrase used for disease acquired by the patient under medical care. They represent for the most part of adverse events affecting hospitalized patients, resulting in increased morbidity, mortality, longer hospital stay, and disability. In the European Union, the annual number of hospital-acquired infections was about 4.5 million resulting in almost 37,000 deaths and 16 million extra days at the hospital per year (Sax et al. 2013).

Bacterial pathogens account for the greater part of nosocomial infections. In addition, usual monitoring also revealed $5 \%$ of the infections to be caused by viruses. Fungal parasites also act as opportunistic pathogens causing nosocomial infections in immune-compromised individuals (Khan et al. 2017). These healthcare-associated infections encompass central line-associated bloodstream infections, catheter-associated infections, and ventilator-associated pneumonia. They may also occur at the surgery sites, known as surgical site infections.

Hospital epidemiology and infection control data anticipated catheter-related bloodstream infections as the leading HAI, together with urinary tract infections, surgical site infections, and ventilator-associated pneumonia (Mayhall, 2012). The possibility of acquiring these infections is significant in the intensive care units (ICUs), where the World Health Organization (WHO) estimates that roughly $30 \%$ of the patients are affected with (HAls) with associated morbidity and mortality (Allegranzi et al. 2011).

Infection prevention and control (IPC) programme help minimize these HAls. In spite of many initiatives taken for infection prevention and control in India, the successful implementation of these programme has been facing some significant challenges, including inadequate funds, human resources, low nurse-to-patient ratios even in intensive care units (Swaminathan et al. 2017). Nonetheless, data from a small number of facilities in India propose that the implementation of hospital infection measures are feasible and can help reduce infection rates (Jaggi et al. 2013,
Mehta et al. 2013).

Hospital infection control relies both on the physician understanding of epidemiologic observations in intensive care units and their knowledge of findings in the microbiology laboratory. Effective communication and support involving microbiologists, hospital epidemiologists, and clinical infectious diseases specialists are decisive for successful infection control (Ebnother et al. 2008). Long-term implementation of these recommended procedures will require rigorous efforts to reinforce infection prevention and control capacity among staff in healthcare settings.

Keeping in mind the mortality, morbidity rate, length of stay in the hospital and the cost, infection control measures should be implemented to make hospitals as safe as possible by preventing such infections. Hence the present study involved implementation and monitoring of infection control practices to control nosocomial infections in our hospital.

\section{MATERIAL AND METHODS}

Infection control is the discipline concerned with the prevention of nosocomial or healthcare-associated infection. Care hospital, Hyderabad has been a pioneering institution for infection control in our country. The present descriptive study involved implementation and monitoring of the infection control practices to control hospital-acquired infections from 20102017.

\section{Ethical approval}

Ethical approval was obtained from the Institutional Ethics Committee, Care Group of hospitals.

Agenda for initiating this programme was discussed in the hospital infection control committee meeting. Guidelines were formulated by the infection control team in the year 2010 (Table 1) and were periodically revised each year to allow for better compliance of the infection control measures. These guidelines were not merely developed for the healthcare personnel involved in patient care in wards, and critical areas but for the persons responsible for the control of infections in our hospital.

High-end and reserved antibiotic usage was also streamlined in the hospital as a part of the infection control measure. Prescriptions were 
Table 1. Guidelines formulated by the Infection control committee

\begin{tabular}{ll}
\hline S. No. & \multicolumn{1}{c}{ Guidelines } \\
\hline 1. & Aprons to be removed by all the physicians prior to their entry into the intensive care units (ICU'S) \\
2. & Mandatory use of shoe covers by all staff and visitors before entering the intensive care units \\
3. & Disinfection of the stethoscope using an alcohol swab after examining each patient \\
4. & Dry mops instead of brooms to be used in all the ICU'S by the hospital housekeepers \\
5. & Instead of saline wash, heplocks should be used to flush intravenous (IV) lines \\
6. & Sterile drapes for all the Intubatory procedures \\
7. & Cloth masks and caps to be substituted with disposable caps and masks \\
8. & Provision of N-95 masks for all open cases of tuberculosis \\
9. & Color coding stickers for identification of infected patients \\
10. & Use of spacers for nebulization of patients on ventilators \\
11. & The housekeeping, nursing staff, and technicians in critical areas have to be \\
12. & vaccinated against Hepatitis-B \\
13. & Regular monitoring of fumigation practices in the intensive care units \\
14. & Methicillin-resistant Staphylococcus aureus (MRSA) policy was executed \\
15. & Antifungal painting to protect the exterior surfaces from microbial activity \\
16. & Disinfection of all surgical instruments using Korsolex
\end{tabular}

divided into medical \& surgical and the auditing of those prescriptions was performed to prevent the misuse of antibiotics. Improvement of the hand hygiene helps minimize nosocomial infection as proposed by the World Health Organization (WHO) (Abdellatif et al. 2007). Hence we promoted effective measures to improve hand hygiene by organizing training classes on hand washing practices. Hand washing facilities were provided in all critical care areas and nursing stations.

Protocols for appropriate disinfection of patient care equipment were circulated to all nursing stations. Separate places were identified for washing of dressing sets in all the nursing stations before sending it to the Central sterile service department (CSSD). Washing and packing of surgical intensive care units (SICU) dressing sets was implemented in CSSD. Multivariable (MVI) indicators helped in the further assessment of the proper sterilization of the dressing sets.

Apart from this, we conducted regular monthly programs on infection control practices to improve awareness among the physicians, nursing, and hospital housekeepers. Classes on Foley's catheterization addressing the inappropriate catheter use and care of intravascular catheters were given to all nursing stations. Display boards as "wear shoe covers" and "remove white coats" were placed in all ICU'S. Hygienic practices in the canteen were monitored to minimize the transmission of infections to the patients.

Data regarding the observed infection rates were collected by active surveillance from the infection control nurses on daily basis and was documented in the surveillance format. This data from the intensive care units i.e. neonatal intensive care unit (NICU), neurosurgery intensive care unit (NSICU), intermediate care unit (IMCU), intensive care cardiac unit (ICCU), medical intensive care unit (MICU), surgical intensive care unit (SICU), and cardiothoracic intensive care unit (CTICU) was subjected to regular analysis.

Infection control nurse records all the positive cultures from the hospital register following which she obtains further data regarding the admission of the patient from the respective wards. This data regarding the date of admission provides information on the total patient days to help calculate the infection rates. The overall infection rate was calculated by dividing infection episodes by total patient days, multiplied by 1000 .

\section{Statistical data}

Data was compiled using Microsoft excel sheet. Descriptive statistics were used to calculate the infection rates in terms of percentages.

\section{RESULTS}

This study was performed in a tertiary level care hospital, Hyderabad with the primary purpose of reducing the nosocomial infection 
rate i.e. ventilator-associated pneumonia rate, catheter-associated UTI rate, bloodstream, and surgical site infection rate.

The observed nosocomial infection rate in the year 2010 was $26.5 \%$ (Fig. 1). There was no major decrease in the infection rates in the years 2011 and 2012. But over a period of time increased compliance is seen accounting for a decrease of
$8.82 \%$ in the year 2017 . Nosocomial infection rates were further categorized as per the infection site and analyzed as ventilator-associated pneumonia rate, catheter-associated urinary tract infection (UTI) rate, bloodstream infection rate and surgical site infection rate in the present study.

The average ventilator associated pneumonia rate recorded in the year 2010 was

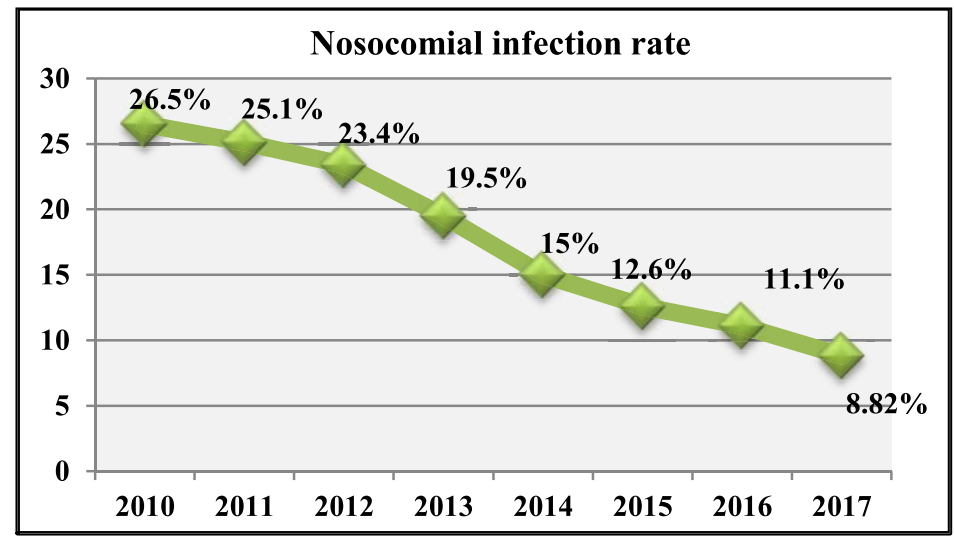

Fig. 1. Nosocomial infection rate from 2010-2017

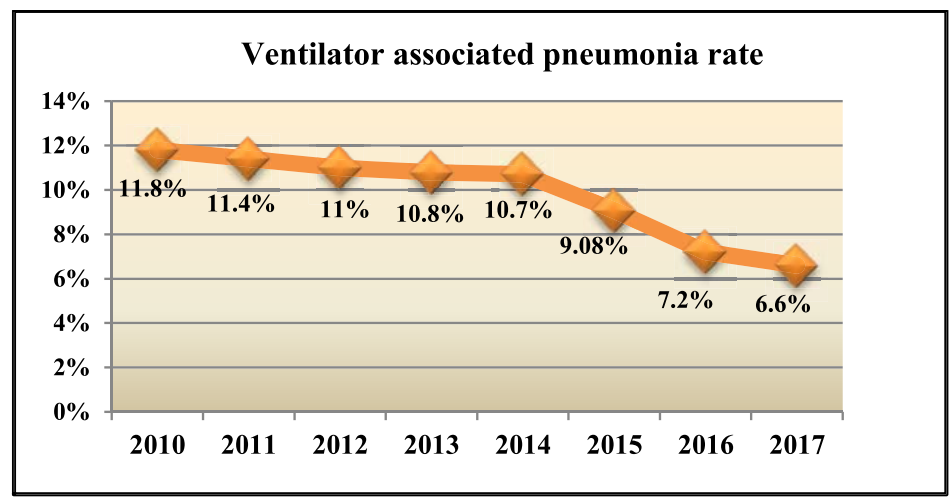

Fig. 2. Ventilator-associated pneumonia rate from 2010-2017

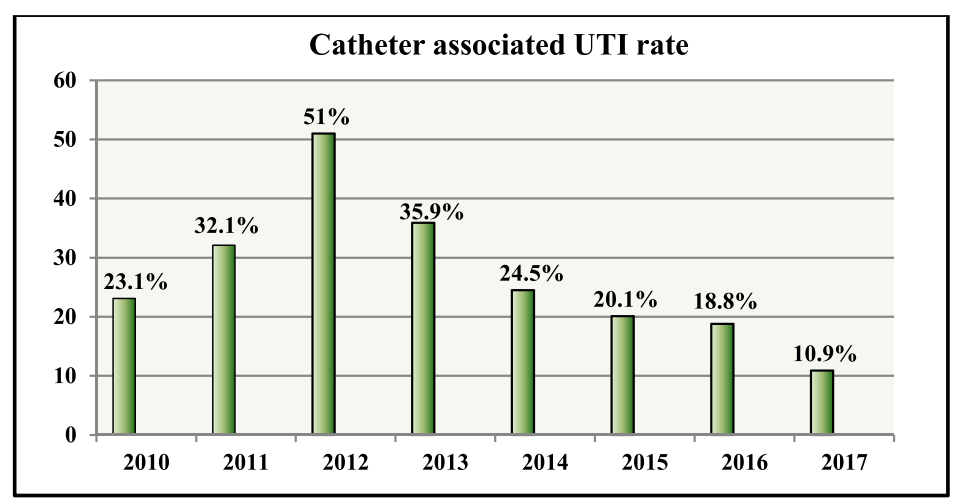

Fig. 3. Catheter-associated urinary tract infection (UTI) rate 
$11.8 \%$ (Fig. 2). Further fallow up years was with $11.4,11,10.8,10.7,9.08,7.2$ and $6.6 \%$ observed rates from 2011 to 2017.

As can be interpreted from the Fig. 3, catheter-associated infection rates decreased from $23.1 \%$ in the year 2010 to $10.9 \%$ in the year 2017 , a decrease of about $12.2 \%$ over a period of 7 years. Bloodstream infection rate reduced from
$12.4 \%$ in the year 2010 to $1.9 \%$ in the year 2017 (Fig. 4). When we compare different categories of nosocomial infection, bloodstream infection elicited the highest reduction.

Surgical site infection rate in the year 2010 was $8.5 \%$. There was a consequent decrease in the infection rates over a period of time. The observed rate in the year 2017 was $2.8 \%$ (Fig. 5).

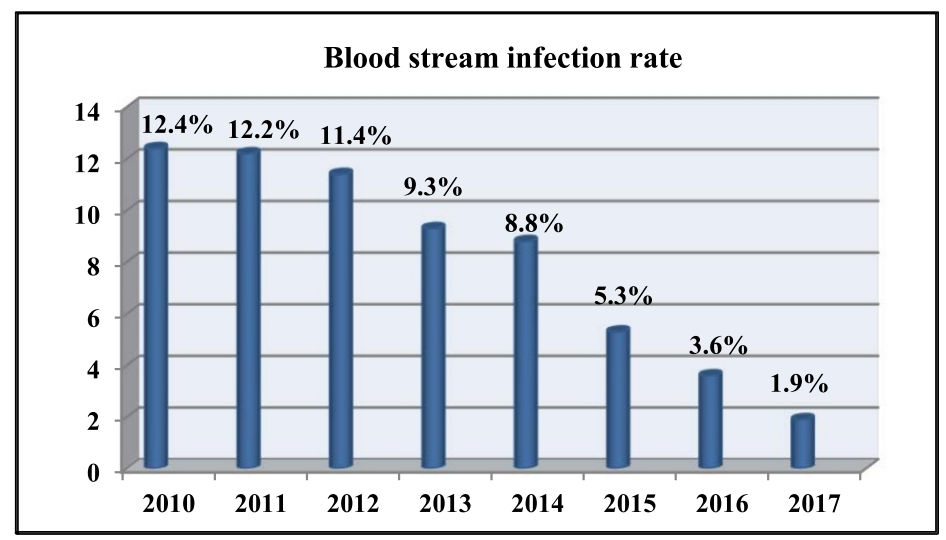

Fig. 4. Observed bloodstream infection rate in the hospital

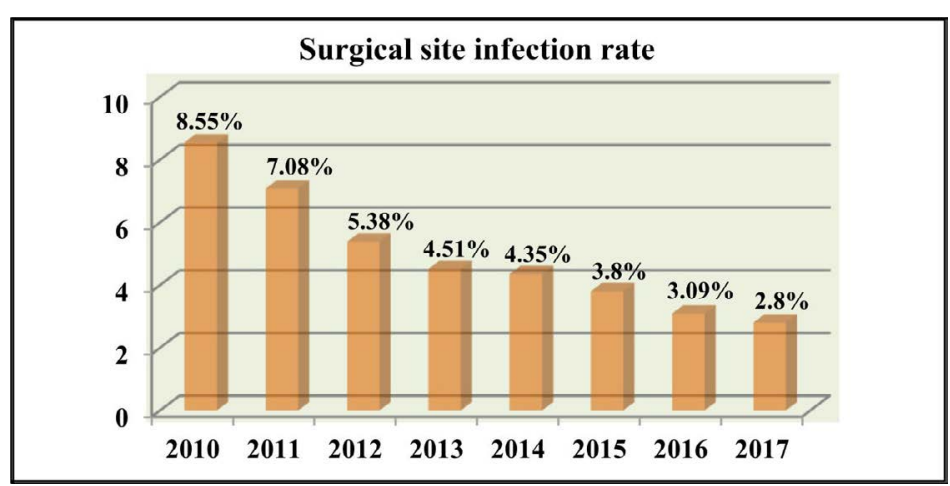

Fig. 5. Observed surgical site infection rate in the hospitals

\section{DISCUSSION}

Hospital-acquired infections are a widespread healthcare problem. It poses a considerable burden particularly in the developing countries where surveillance and research are limited. Nevertheless, basic infection control strategies in these settings can have maximum impact. In the present study, an infection rate of $26.5 \%$ was noted in the year 2010 which reduced to $8.82 \%$ over a period of 7 years. Ebnother, et al. before and after implementation of an infection control program found a similar reduction in infection rates from 11.7 to $6.8 \%$ (Ebnother et al. 2008). This reduction in nosocomial infection rates in the current study could be attributed to improved hand hygiene, mandatory use of shoe covers and removal of aprons prior to the entry into the intensive care units, appropriate disinfection of patient care equipments, regular monitoring of fumigation practices in the intensive care units, and the various other formulated infection control strategies by our infection control team. 
Nosocomial infection rates were additionally analyzed as per the infection site; ventilator-associated pneumonia rate, catheterassociated urinary tract infection (UTI) rate, bloodstream infection rate and surgical site infection rate in the present study. Ventilatorassociated pneumonia (VAP), the second most common nosocomial infection in the intensive care unit is pneumonia that develops $48-72$ hours after mechanical ventilation resulting from the invasion of the lower respiratory tract and lung parenchyma by microorganisms. It is usually attributed to antibiotic-sensitive pathogens and multidrugresistant bacteria (Kalanuria et al. 2014).

In the present study, the average ventilator-associated pneumonia rate recorded in the year 2010 was $11.8 \%$ which reduced to $6.6 \%$ in the year 2017. Infection control practices in an Italian intensive care unit also found reduced ventilator-associated pneumonia rate which was in line with our study (Prospero et al. 2008). Streamlining high end and reserved antibiotic prescriptions, use of spacers to nebulize patients on ventilators, implementation of MRSA policy accounted for the decrease in VAP infection rates in our study. Additionally, rigorous hand hygiene and environmental cleaning practices limited the spread of pathogens from patient-to-patient via healthcare workers and equipment.

Catheter-associated UTI (CAUTI) represents the third most common type of infections occurring in the intensive care after pneumonia and lower respiratory tract infections (Lee et al. 2013). Catheter-associated infection rates decreased from $23.1 \%$ in the year 2010 to $10.9 \%$ in the year 2017 , a decrease of about $12.2 \%$ over a period of 7 years in the present study. A multidimensional infection control approach on catheter-associated UTI rates in an intensive care unit in Lebanon used a bundle of infection control interventions and reported a significant reduction in the infection rates which was very much in accordance with our study (Kanj et al. 2013). The most frequent urinary catheter in use is the Foley indwelling urethral catheter, a closed sterile system comprising of a tube inserted through the urethra and held in place by an inflatable balloon to allow urinary drainage of the bladder (Jacobsen et al. 2008). Literature has consistently linked inappropriate catheter use with the development of CAUTI (Topal et al. 2005). The classes conducted on Foley catheterization addressing the care of catheters and removal of them when no longer in need, helped control the nosocomial CAUTI rate in the present study.

One possible cause for nosocomial bloodstream infections is because of the antibioticresistant organisms (Wisplinghoff, 2004). The prevalence has also found to be correlated with the frequency of use of intravascular catheters (Jarvis et al. 1996). A $10.5 \%$ reduction in the bloodstream infection was noted in the present study similar to the effect of an infection control program, using education and performance feedback on intensive care units, for central line-associated bloodstream infection (BSI) (Leblebicioglu et al. 2013). Analysis of MDR pathogens sensitivity resistance pattern by the infection control team and care of intravascular catheters accounted for this reduced bloodstream infection rate.

Addressing the last parameter i.e. surgical site, a $5.7 \%$ decrease noted in our study could be attributed to the prophylactic antibiotic administration protocols, and the operating room environment i.e. regular monitoring of fumigation practices, and implementation of air sampling in the intensive care units.

\section{CONCLUSION}

In conclusion, our study revealed that by means of surveillance, hospitals can devise strategies to control inappropriate infection control practices in the intensive care units, and the wards. These guidelines help control the nosocomial infection rates thereby reducing the hospital costs, total patient days and the mortality rate. It has been shown that a coordinated infection control approach which includes hand hygiene, appropriate skin preparation, and catheter line care, and removal of lines when they are no longer needed can significantly impact the occurrence of these HAls.

\section{ACKNOWLEDGEMENT}

None

\section{CONFLICT OF INTEREST}

The authors declare that there is no conflict of interest. 


\section{REFERENCES}

1. Sax H, Clack L, Touveneau S, Jantarada F da L, Pittet $D$, Zingg W. Implementation of infection control best practice in intensive care units throughout Europe: a mixed-method evaluation study. Implementation Science: IS. 2013; 8:24.

2. Khan HA, Baig FK, Mehboob R. Nosocomial infections: Epidemiology, prevention, control and surveillance. Asian Pacific Journal of Tropical Biomedicine., 2017; 7(5):478-82.

3. Mayhall CG. Hospital Epidemiology and Infection Control. 4. Philadelphia, PA, USA: Lippincott Williams \& Wilkins; 2012.

4. Allegranzi B, Bagheri Nejad S, Chraiti MN, Combescure C, Attar H, Pittet D. Report on the Burden of Endemic Health Care-Associated Infection Worldwide. Geneva, Switzerland: World Health Organization; 2011.

5. Swaminathan S, Prasad J, Dhariwal AC, Guleria R, Misra MC, Malhotra R, Mathur P, Walia K, Gupta S, Sharma A, Ohri V. Strengthening infection prevention and control and systematic surveillance of healthcare associated infections in India. bmj., 2017; 358:j3768.

6. Jaggi N, Rodrigues $C$, Rosenthal VD, et al. Impact of an international nosocomial infection control consortium multidimensional approach on central line-associated bloodstream infection rates in adult intensive care units in eight cities in India. Int. J. Infect. Dis., 2013; 358.

7. Mehta $\mathrm{Y}$, Jaggi N, Rosenthal VD, et al. Effectiveness of a multidimensional approach for prevention of ventilator-associated pneumonia in 21 adult intensivecare units from 10 cities in India: findings of the International Nosocomial Infection Control Consortium (INICC). Epidemiol Infect., 2013; 358:2483-91

8. Ebnother C, Tanner B, Schmid F, La Rocca V, Heinzer I, Bregenzer T. Impact of an infection control program on the prevalence of nosocomial infections at a tertiary care center in Switzerland. Infection Control \& Hospital Epidemiology., 2008; 29(1):38-43.

9. Abdellatif $A$, Bagian JP, Barajas $E R$, Cohen $M$, Cousins $D$, Denham CR, Essinger K, Gegelashvili G, Glenister H, Hoffman C, Horvath D. Improved Hand Hygiene to Prevent Health Care-Associated Infections: Patient Safety Solutions, Volume 1, Solution 9, May 2007. Joint Commission Journal on Quality and Patient Safety, 2007; 33(7):460-3.

10. Kalanuria AA, Zai W, Mirski M. Ventilator-associated pneumonia in the ICU. Critical Care. 2014;18(2):208. doi:10.1186/cc13775.

11. Prospero E, Bacelli $S$, Barbadoro $P$, Nataloni $S$, D'errico MM, Pelaia P, SICU Working Group. Improvement of the ventilator associated pneumonia rate with infection control practices in an Italian ICU. Minerva Anestesiol., 2008; 74(10):537-41.

12. Lee JH, Kim SW, Yoon BI, Ha U-S, Sohn DW, Cho Y-H. Factors That Affect Nosocomial CatheterAssociated Urinary Tract Infection in Intensive Care Units: 2-Year Experience at a Single Center. Korean Journal of Urology, 2013; 54(1):59-65. doi:10.4111/ kju.2013.54.1.59.

13. Kanj SS, Zahreddine N, Rosenthal VD, Alamuddin L, Kanafani Z, Molaeb B. Impact of a multidimensional infection control approach on catheter-associated urinary tract infection rates in an adult intensive care unit in Lebanon: International Nosocomial Infection Control Consortium (INICC) findings. International Journal of Infectious Diseases, 2013;17(9):e686-90.

14. Jacobsen SM, Stickler DJ, Mobley HLT, Shirtliff ME. Complicated Catheter-Associated Urinary Tract Infections Due to Escherichia coli and Proteus mirabilis. Clinical Microbiology Reviews, 2008; 21(1):26-59. doi:10.1128/CMR.00019-07.

15. Topal J, Conklin S, Camp K, Morris V, Balcezak T, Herbert P. Prevention of nosocomial catheter-associated urinary tract infections through computerized feedback to physicians and a nurse-directed protocol. American Journal of Medical Quality, 2005; 20(3):1216.

16. Wisplinghoff $H$, Bischoff $T$, Tallent SM, Seifert $H$, Wenzel RP, Edmond MB. Nosocomial bloodstream infections in US hospitals: analysis of 24,179 cases from a prospective nationwide surveillance study. Clinical infectious diseases, 2004; 39(3):309-17.

17. Jarvis WR, Cookson ST, Robles MB. Prevention of nosocomial bloodstream infections: a national and international priority. Infection Control \& Hospital Epidemiology. 1996;17(5):272-5.

18. Leblebicioglu $H$, Ozturk R, Rosenthal VD, Akan OA, Sirmatel F, Ozdemir D, Uzun C, Turgut H, Ersoz G, Koksal I, Ozgultekin A. Impact of a multidimensional infection control approach on central line-associated bloodstream infections rates in adult intensive care units of 8 cities of Turkey: findings of the International Nosocomial Infection Control Consortium (INICC). Annals of clinical microbiology and antimicrobials, 2013; 12(1):10. 